Journal of Pediatric Gastroenterology and Nutrition, Publish Ahead of Print

DOI : 10.1097/MPG.0000000000002802

\title{
Safety of Thiopurine Use in Paediatric Gastrointestinal Disease
}

Erasmo Miele ${ }^{1}$, Marc A Benninga ${ }^{2}$, Ilse Broekaert ${ }^{3}$, Jernej Dolinsek ${ }^{4}$, Emmanuel Mas $^{5}$, Rok Orel $^{6}$, Corina Pienar ${ }^{7}$, Carmen Ribes-Koninckx ${ }^{8}$, Rut Ann Thomassen ${ }^{9}$, Mike Thomson $^{10}$, Christos Tzivinikos ${ }^{11}$, and Nikhil Thapar $^{12,13}$

${ }^{1}$ Department of Translational Medical Science, Section of Paediatrics, University of Naples "Federico II", Italy

${ }^{2}$ Department of Paediatric Gastroenterology, Hepatology and Nutrition, Emma Children's Hospital/Amsterdam UMC

${ }^{3}$ University of Cologne, Faculty of Medicine and University Hospital Cologne, Department of Pediatrics, Cologne, Germany

${ }^{4}$ University Medical Centre Maribor, Department of Pediatrics, Gastroenterology unit, Ljubljanska ulica 5, Maribor, Slovenia and Medical faculty of University of Maribor, Department of Paediatrics, Taborska ulica 8 Maribor, Slovenia ${ }^{5}$ IRSD, Université de Toulouse, INSERM, INRA, ENVT, UPS, Toulouse, France and Unité de Gastroentérologie, Hépatologie, Nutrition, Diabétologie et Maladies Héréditaires du Métabolisme, Hôpital des Enfants, CHU de Toulouse, F-31300, France

${ }^{6}$ Department of Gastroenterology, Hepatology and Nutrition, Children's Hospital, University Medical Centre Ljubljana, Ljubljana 1000, Slovenia

${ }^{7}$ Paediatrics Department "Victor Babes" University of Medicine and Pharmacy Timisoara, Romania 
${ }^{8}$ Pediatric Gastroenterolgy, Hepatology and Nutrition, La Fe University Hospital, Valencia, Spain

${ }^{9}$ Pediatric Nutrition and Dietetics Unit, Dept. of Paediatric and Adolescent Medicine, Oslo University Hospital, Norway

${ }^{10}$ Department of Paediatric Gastroenterology, Sheffield Children's NHS Foundation Trust. Sheffield

${ }^{11}$ Department of Paediatric Gastroenterology, Hepatology and Nutrition, Alder Hey Children's Hospital, Liverpool, UK

${ }^{12}$ Neurogastroenterology and Motility Unit, UCL Great Ormond Street Institute for Child Health and Great Ormond Street Hospital, London, United Kingdom

${ }^{13}$ Current Address: Gastroenterology, Hepatology and Liver Transplant, Queensland Children's Hospital, Brisbane, Australia.

\section{Corresponding Authors}

Erasmo Miele, MD, PhD

Department of Translational Medical Science, Section of Paediatrics

University of Naples “Federico II", Via S. Pansini, 5, 80127 Naples Italy

Email: erasmo.miele@unina.it

\section{Nikhil Thapar, BM FRCPCH PhD}

UCL Great Ormond Street Institute for Child Health and Great Ormond Street Hospital, Great Ormond Street, London United Kingdom, WC1N 3JH

Current: Queensland Children's Hospital, Stanley Street, Brisbane, Australia, QLD 4101

Email: nikhil.Thapar@health.qld.gov.au 


\begin{abstract}
Thiopurines, alone or in combination with other agents, have a pivotal role in the treatment of specific gastrointestinal and hepatological disorders. In inflammatory bowel disease and autoimmune hepatitis thiopurines have proven their value as steroid sparing agents for the maintenance of remission and may be considered for preventing postoperative Crohn's disease recurrence where there is moderate risk of this occuring. Their use with infliximab therapy reduces antibody formation and increases biologic drug levels. However, the routine clinical use of thiopurines has been questioned due to a number of potential adverse effects. The aim of this paper is to provide information regarding the use, and in particular, safety of these agents in clinical practice in the light of such potentially severe, albeit rare, effects.
\end{abstract}

Keywords: Thiopurines, azathioprine, 6-mercaptopurine, inflammatory bowel disease, autoimmune hepatitis, adverse effects

\title{
Learning Points
}

- In inflammatory bowel disease and autoimmune hepatitis, albeit with some debatable evidence, thiopurines have proven their value, as steroid sparing agents for the maintenance of remission.

- Thiopurines may offer distinct advantages either as monotherapy or in combination with other drugs, such as biologicals, in which they may enhance therapeutic effects by reducing antibody formation and increasing drug levels.

- Despite the efficacy of thiopurines, the wider use of several more effective biologic agents makes their use more questionable especially given safety concerns, which must to be taken into account. 


\section{Introduction}

Thiopurines are a class of immunosuppressant drugs that include azathioprine (AZA), 6mercaptopurine (6-MP), and 6-thioguanine (6-TG) (1).

In children, thiopurines, alone or in combination with other agents, are used in specific gastrointestinal disorders, especially inflammatory bowel disease (IBD) and autoimmune hepatitis (AIH), as well as in haematological malignancies, solid organ transplantation and several auto-immune disorders (2).

Despite evidence for the usefulness of thiopurines, as a steroid sparing tool, in ulcerative colitis (UC) and in AIH, their efficacy in Crohn's disease (CD) is more questionable and safety concerns with regards to this modality must to be taken into account when deciding on therapy (3). Indeed, given the ongoing debate regarding efficacy and safety of these agents, there is variability in how they are used for clinical therapy in IBD children. This is reflected in differences in clinical practice between North American paediatric gastroenterologists, who prefer not to use thiopurines in IBD children given a perception of low effectiveness/high risk, and their colleagues in Europe/Southern Hemisphere, who continue to use thiopurines as monotherapy, supported by current clinical guidelines (4). This paper offers information regarding the use of these agents in clinical practice in the light of their potentially severe, albeit rare, adverse effects.

\section{Mechanism of Action}

Thiopurines are prodrugs and must be converted intracellularly to 6-thioguanine nucleotides (6-TGNs) to exert their therapeutic effect. After oral intake, AZA is rapidly converted, predominantly by glutathione-S-transferase, to 6-MP. 6-MP can then be metabolised via three competing pathways: xanthine oxidase (XO), thiopurine methyltransferase (TPMT), and hypoxanthine guanine phosphoribosyltransferase (HGPRT). The complete metabolism of 
thiopurines leading to the production of 6TGNs and their mechanism of action are illustrated in Figure 1. (5). 6-TGNs are antimetabolites, purine analogues that are incorporated into nucleic acids resulting in inhibition of nucleotide and protein synthesis and ultimately in inhibition of lymphocyte proliferation (6). Further mechanisms of action include inhibition of several genes involved in intestinal inflammation and trafficking of leukocytes to the gut, such as tumor necrosis factor (TNF)-related apoptosis-inducing ligand (TRAIL), TNF receptor superfamily member 7 (TNFRS7), and alpha-4-integrin in the presence of T-cell activation (7). In addition, it has been proposed that T-cell apoptosis may be induced by blockage of the CD28-dependent GTPase Ras-related C3 botulinum toxin substrate 1 (Rac1) activation pathway. The suppression of Rac1-target genes, such as mitogen-activated protein kinase, $N F-\mathrm{k} B$ and $b c l-x(L)$, causes mitochondrial apoptosis (8).

\section{Indications}

Thiopurines have been used for decades in the therapeutic armamentarium of paediatric and adult IBD, as the first-choice drug of second-line maintenance therapy. Nevertheless, this initial indication has been progressively challenged by the introduction and wider use of several biologic agents. However, AZA or 6-MP are still currently recommended as one option for steroid free remission maintenance in children with $\mathrm{CD}$ at risk for poor disease outcome and for maintaining remission in children with UC who are corticosteroid-dependent or relapsing frequently ( $\geq 2$ relapses per year) despite optimal 5-ASA treatment or 5-ASA intolerance $(9,10)$. Nevertheless, due to the safety concerns, the recent guideline of the Canadian Association of Gastroenterology for the medical management of paediatric CD, suggests a thiopurine can be used in female patients to maintain remission, given that the evidence suggests that the benefits may outweigh the risks in this gender (11). In addition, thiopurines may also be considered following an episode of acute severe colitis and to 
prevent postoperative relapse in children with a moderate risk of $\mathrm{CD}$ recurrence $(12,13)$. However, their efficacy in preventing postoperative recurrence is debatable with all the available data coming only from adult studies $(14,15)$. In addition, where thiopurines have failed preoperatively their postoperative use requires careful risk-benefit analysis (13).

Thiopurines used alone do not induce remission in CD and UC but have a role in IBD maintenance therapy (9-11). Recently, a prospective cohort study on 129 children with mildmoderate IBD, showed that thiopurines were both safe and effective in achieving steroid and exclusive enteral nutrition-free remission; this was achievedwithout treatment escalation by 12 months, in $21 \%$ and $27 \%$ of the children with CD and UC, respectively, especially in those with lower baseline disease activity (16). It should be noted, however, that the studied cohort had no potential risk factors for a complicated IBD disease course and most of the children were exposed to drug dose optimization and drug levels monitoring (16).

The superiority of combination therapy, AZA with infliximab (IFX), over IFX monotherapy has been demonstrated in adult and paediatric patients with IBD. Indeed, combination therapy was associated with higher IFX trough levels and reduced formation of IFX antibodies than monotherapy, which is likely contribute to its greater efficacy (17-23). In contrast to previous studies, in paediatric and adult IFX-treated patients with active luminal CD the PANTS study showed that thiopurines reduced immunogenicity in patients in a dose-dependent manner without an obvious threshold effect (23-25). In addition, while the post-hoc analyses of the SONIC study demonstrated that the primary effect of AZA was on pharmacokinetics of IFX, the PANTS study suggested that concomitant immunomodulator use in IFX-treated patients was associated with higher week 54 remission compared with no immunomodulator use, an effect which was independent of drug concentration or immunogenicity status, suggesting possible additional benefits of immunosuppression to anti-TNF therapy $(25,26)$. 
AZA in combination with prednisone also remains the mainstay of treatment for autoimmune hepatitis $(\mathrm{AIH})$ with several reports showing high remission rates and favourable outcomes in both adult and juvenile AIH (27). AZA monotherapy, albeit unsuccessful in the induction of remission, is effective both in adults and in children as maintenance therapy in AIH (28). The use of AZA with prednisone is included in the recent position statement on the diagnosis and management of paediatric autoimmune liver disease by the Hepatology Committee of the European Society for Paediatric Gastroenterology, Hepatology and Nutrition (ESPGHAN) (29).

\section{Optimising Thiopurine Therapy}

In patients with normal TPMT activity, the thiopurine dose should be approximately 2 to 2.5 $\mathrm{mg} / \mathrm{kg}$ for AZA and 1 to $1.5 \mathrm{mg} / \mathrm{kg}$ for MP, taken as a single daily dose, with or after food (30). Children younger than 6 years may require higher doses of AZA per kg of body weight with doses of up to $3 \mathrm{mg} / \mathrm{kg} /$ day (31).

Measuring thiopurine metabolites may help in further dose adjustments and to reduce adverse events, while considering 6-TGN levels (measured using the method of Lennard et al) of 230 to $450 \mathrm{pmol} / 8 \times 10^{8} \mathrm{RBCs}$ and 6-MMP levels $<5700 \mathrm{pmol} / 8 \times 10^{8} \mathrm{RBCs}$ as optimal $(32,33)$. A therapeutic effect is usually evident after 8 to 14 weeks of treatment (9). Recently, in adults, to reduce the increased risk of side effects of the combination therapy (AZA with IFX), a dose reduction of AZA was suggested in order to achieve a threshold 6-TGN level (measured using the method of Lennard et al.) not lower than $105 \mathrm{pmol} / 8 \times 10^{8} \mathrm{RBCs}(23,32)$. Neither 6-TGN nor 6-MMP concentrations, however, could completely predict the onset of myelotoxicity and hepatotoxicity, thus, regular monitoring of blood counts and liver enzymes is recommended (9). Although an ideal therapeutic level for use in AIH has not been determined, $87 \%$ of 66 children with $\mathrm{AIH}$ in a recent retrospective series were reported to 
maintain sustained biochemical remission (normal transaminase levels) in association with

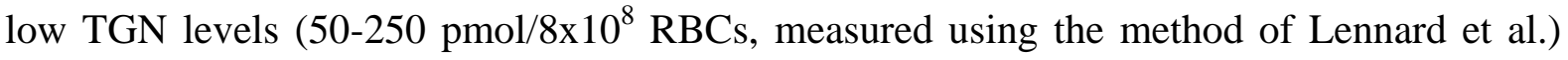
$(28,32)$. Recently, using easily determined laboratory parameters (e.g. mean corpuscular volume, red blood cell distribution width, S-albumin, hematocrit, mean corpuscular hemoglobin concentration, platelet count, serum iron, transferrin saturation) and including age, and sex, models have been proposed in children, as an alternative to the more expensive thioguanine metabolite monitoring, for predicting patients with low 6-TGN and AZA treatment non-adherence. Nevertheless, these models cannot fully substitute standard measurement of intracellular metabolites in erythrocytes $(34,35)$. However, in a large paediatric population, proactive thiopurine metabolite monitoring was not shown to impact on the durability of thiopurine monotherapy. Furthermore, 6-month steroid-free clinical remission and 6-TGN levels were not different between a standardised, weight-based dosing strategy and a metabolite-driven, optimized dosing strategy (36).

Methylation of MP by TPMT has a critical role in thiopurine metabolism, given the side effects of thiopurine drugs correlate with the accumulation of high levels of TGNs. Population-based studies have shown a trimodal distribution of TPMT activity, with $89 \%$ of the population having normal or high (homozygous), $11 \%$ having intermediate (heterozygotes), and $0.3 \%$ having minimal enzyme activity (37).

Individuals with complete TPMT deficiency who receive standard doses of AZA or MP are highly likely to develop severe and potentially fatal myelosuppression. Furthermore, TPMT activity changes with age with newborn and younger children possessing higher enzyme activity compared to older children and adults (38). Therefore, a TPMT assay (either phenotype or genotype) prior to starting thiopurines, as a means to prevent life-threatening leukopenia in patients with low enzyme activity without increasing overall healthcare costs, is recommended by the American Gastroenterological Association and encouraged in the 
recent paediatric UC guidelines from the European Crohn's and Colitis Organisation and ESPGHAN (10, 39-41). However, some studies suggest that most patients who developed leukopenia did not have mutant TPMT alleles, and that routine blood count monitoring remains essential especially during initiation of treatment (10). In addition, as for the other key enzymes in thiopurine metabolism, nucleoside diphosphate-linked moiety X-type motif (NUDT15), catalysing the dephosphorylation of the 6-thioguanine triphosphate metabolites to the 6-thioguanine monophosphate (TGMP), prevents the incorporation of thiopurine active metabolites into DNA. In case of NUDT15 genetic defects, recent evidence suggests that the increase in the TGMP availability for incorporation into the DNA leads to an elevated risk of clinical toxicity. Thus, it has been suggested that the NUDT15 gene be considered a biomarker for thiopurine dose adjustment, significantly contributing towards further reducing the incidence of potentially lethal adverse drug reactions $(42,43)$

\section{Safety}

Despite the efficacy of thiopurines as steroid sparing tools in UC and AIH, they have a relatively narrow therapeutic index. Adverse reactions occur in $10-28 \%$ of patients, including gastrointestinal intolerance, pancreatitis, hypersensitivity, and life-threatening bone marrow suppression, which often results in the withdrawal of treatment $(44,45)$ (Table 1). In adults with UC receiving thiopurines, a relative risk of 2.82 for serious adverse events has been reported (46). A thiopurine withdrawal rate due to adverse events has been reported in 2-30\% of children $(15,47,48)$.

Among dose-independent minor side effects, rash, arthralgias, nausea, vomiting, diarrhoea and flu-like reactions represent common manifestations in patients receiving AZA or 6-MP (30). 
Advising patients to "switch" to another thiopurine (AZA to 6-MP and vice versa), to split the dose, to use it prior to bedtime or with food are alternative strategies that may reduce nausea (49). The metabolites of the thiopurines are responsible for their therapeutic effect as well as some of their adverse effects. Higher levels of 6TG are associated with bone marrow suppression, whereas high levels of 6MMP are associated with hepatotoxicity (50).

Major adverse effects reported for thiopurines are pancreatitis, neutropenia, hepatotoxicity and malignancy.

\section{Pancreatitis}

Pancreatitis occurs in approximately $4 \%$ of patients treated with thiopurines, usually within weeks of beginning treatment, and is considered an idiosyncratic, dose-independent drug reaction (51). AZA-induced pancreatitis is more prevalent in children with $\mathrm{CD}(4.9 \%)$ than in children with UC (1.1\%) or with autoimmune hepatitis (1.5\%) (52). Recently, in a SwedishDanish cohort of children with IBD, the use of AZA was associated with a six-fold elevated risk of acute pancreatitis within 90 days following treatment initiation (53). Switching thiopurine (AZA to 6-MP and vice versa) in the case of pancreatitis has traditionally not been recommended but evidence has challenged this opinion (49).

\section{Bone Marrow Toxicity}

Mild leukopenia $\left(3.0-4.0 \times 10^{9} / \mathrm{L}\right)$ is the most common haematological side effect occurring with standard doses $(2.0 \mathrm{mg} / \mathrm{kg})$ of AZA. In large adult patient cohorts, the frequency of leukopenia ranges from about 2 to $15 \%$, depending on its definition (2). In children, leukopenia has been reported in about $10 \%$ of children monitored every 3 months during treatment with AZA or 6-MP and resolves without sequelae, either spontaneously or with dose reduction or drug discontinuation (48). However, severe myelosuppression is the most 
common serious, occasionally fatal, adverse event of treatment with AZA. Severe myelotoxicity is more likely to occur in patients with absent or decreased TPMT activity, although factors unrelated to TPMT activity may predispose patients to myelotoxicity (2).

\section{Risk of Infection}

Thiopurine therapy has been also associated with an increased rate of serious infection, even in the absence of neutropenia (50). In addition, adult patients receiving thiopurines have increased risk of developing opportunistic infections (odd ratio 3.1; 95\% CI: 1.7-5.5) (41) and when compared to anti-TNF monotherapy, combination therapy is associated with increased risks of serious infections [hazard ratio (HR), 1.23; 95\% CI, 1.05-1.45] (54). Nevertheless, compared with thiopurine monotherapy, anti-TNF monotherapy is associated with increased risks of serious opportunistic bacterial and mycobacterial infection (HR, 1.71; 95\% CI, 1.56-1.88) (54). Conversely, anti-TNF monotherapy was associated with decreased risk of opportunistic viral infection compared to thiopurine monotherapy $(\mathrm{HR}, 0.57,95 \% \mathrm{CI}$, $0.38-0.87$ ), suggesting that this risk in the context of combination therapy is driven by thiopurines (55).

In contrast, a post hoc analysis on 188 children with $\mathrm{CD}$ showed that the rate of infectious adverse events is similar in patients receiving adalimumab monotherapy $(n=71)$ compared to immunomodulator, and adalimumab combination therapy [AZA: $n=56 / 188$ (30\%); mercaptopurine: $\mathrm{n}=37 / 188(20 \%)$ and methotrexate: $\mathrm{n}=24 / 188(13 \%)$ ]. However, the sample size of the study was too limited to drive definitive conclusions (56).

\section{Hepatotoxicity}

Patients receiving thiopurines may present with mild elevations in transaminases that are transient or reversible with dose reduction, as well as, albeit rarely, nodular regenerative 
hyperplasia and portal hypertension, which can be progressive (57). Liver injury is associated with 6MMP levels $>5700 \mathrm{pmol} / 8 \times 10^{8} \mathrm{RBC}$. Elevated 6MMP levels occur more frequently in patients with high TPMT activity that preferentially metabolises thiopurine to 6MMP instead of 6TG. Concomitant use of allopurinol $(50 \mathrm{mg}$ once daily in patients $<30 \mathrm{~kg}$ and $100 \mathrm{mg}$ once daily in patients $>30 \mathrm{~kg}$, maximum $5 \mathrm{mg} / \mathrm{kg}$ ) with a reduced dose of AZA (to approximately $25 \%$ to $30 \%$ of initial dose), may provide a valid therapeutic option in cases of a hyperactive TPMT (10). Recently, low-dose thiopurine-allopurinol (LDTA) combination therapy has been demonstrated as a safe and beneficial optimisation strategy in adult IBD patients. Indeed, although mild myelotoxicity and hepatotoxicity were commonly observed, they rarely required LDTA treatment cessation (58). However, children must be closely monitored given this increased risk of toxicity (10).

\section{Malignancies}

Thiopurines are associated with an increased risk of lymphoma, hemophagocytic lymphohistiocytosis (HLH) and non-melanoma skin cancer (NMSC).

\section{Haematologic malignancies}

It is postulated that chronic lymphopenia caused by thiopurine exposure suppresses the host cellular-mediated immune response via suppressor T-lymphocytes during primary EpsteinBarr virus (EBV) infection; this impaired response leads to hyperproliferation of infected lymphocytes with ensuing complications (50). Thus, seronegativity in patients starting thiopurines has long been a concern (59). Although HLH is very rare, data from the DEVELOP registry, evaluating the risk of malignancy in 5766 paediatric patients with IBD, showed that when limited to primary EBV-associated cases, an incidence rate of $0.2 / 1000$ 
patient-years was observed. Three of the 5 patients, who developed HLH were adolescent females and 1 case of HLH was associated with CMV infection (3). The CESAME study reported that in an adult cohort of almost 20000 adult IBD patients over $52 \%$ of the 23 cases of lymphoproliferative disorders were EBV positive, with an incidence rate of $0.1 / 1000$ patients-years for primary EBV infection-related post-mononucleosis lymphoma, a phenomenon observed in EBV naïve young men less than 35 years of age (60). A more recent Dutch nationwide study confirmed a strong relation between EBV-positive lymphoma and thiopurine use, especially in subjects younger than 50 years (61). These potential risks led the European Crohn's and Colitis Organisation to state that EBV IgG screening should always be considered before initiation of immunomodulatory treatment and that anti-TNFs should be preferentially used in seronegative children (62). Despite these recommendations, a recent cohort study from the Paediatric IBD Porto Group of the ESPGHAN showed that only $21.9 \%$ of children starting AZA were checked for EBV status and among those tested, AZA was started in almost all the cases of seronegative EBV patients, irrespective of gender (63). A meta-analysis found that IBD patients receiving thiopurines had a higher risk of lymphoma compared to the general population, with a standardised incidence ratio (SIR) of 4.92 (95\% CI, 3.10-7.78) (64). A longitudinal surveillance of more than 189.000 IBD adult patients for a median time of 7 years revealed an increased risk of lymphoma among subjects receiving thiopurine monotherapy (adjusted HR, 2.60; 95\% CI, 1.96-3.44; $\mathrm{P}<.001$ ). However, the absolute incidence rate of $0.54(95 \% \mathrm{CI}, 0.41-0.67)$ per 1000 person-years was low among patients exposed to thiopurines. The exposure to thiopurines and/or anti-TNFs resulted in a higher risk in males compared to females (adjusted HR, 1.56; 95\% CI, 1.251.94), with the risk increased in both groups compared to those not receiving thiopurine (65). A meta-analysis of studies on IBD adult patients measured absolute risk, computing the number of patients needed to harm $(\mathrm{NNH})$, which represents the number of patients needed to 
be treated for one year with thiopurine to cause one additional case of lymphoma (64). Assuming a relative risk of lymphoma of 3.0, and using the National Cancer Institute's Surveillance Epidemiology and End Results Database data from 2003-2007, the number of patients needed to cause one additional lymphoma ranges from 6.897 in those aged 20-29, to 513 in those aged $70-79(66,67)$. However, only current thiopurine use was associated with an increased lymphoma risk, potentially justifying withdrawal of immunomodulators as early as possible in paediatric patients (67). Indeed, in children with IBD, ongoing thiopurine exposure or discontinuation of therapy within 1 year of malignancy diagnosis, had a SIR of 4.45 (CI: 1.92, 8.77). Conversely, paediatric IBD patients who discontinued thiopurine therapy for 1 or more years before malignancy diagnosis had a SIR of 1.48 (CI: 0.30, 4.32), similar to that for the thiopurine non-exposed group: 1.30 (CI: $0.16,4.71)$. Therefore, these findings suggest that stopping thiopurines for 1 year or longer may reduce the malignancy risk (3).

Hepatosplenic T-cell lymphoma (HSTCL) is a very rare but fatal complication of thiopurine therapy, especially, in young males. Indeed, 93.5\% of HSTCL, cases were reported in men and only $6.5 \%$ in women $(\mathrm{p}<0.05)(68)$. The paediatric DEVELOP registry reported 2 cases of HSTCL occurring during active thiopurine therapy; neither of the patients with HSTCL were exposed to IFX, adalimumab or methotrexate (3). Moreover, among the other reported malignancies, 9 of 15 events were lymphoid malignancies and the remaining 6 cases included solid tissue malignancies $(n=3)$ and skin malignancies $(n=3)$. Ten patients were exposed to IFX, and among them: 5 patients were also exposed to adalimumab, 6 patients to methotrexate and 9 out of 10 to thiopurines. Four patients were exposed to thiopurines in the absence of biologics. Thus, 13 of the 15 patients with malignancies were exposed to thiopurines and the majority of these patients developed malignancy after less than 5 years of thiopurine exposure. (3). 
More recently, the paediatric IBD Porto group of ESPGHAN reported the data of 43 malignancies. Haematopoietic tumours $(n=21,49 \%)$ were the most frequently reported type of malignancy with a higher risk in IBD patients exposed to thiopurines $(n=20 / 21,95 \%)$. More specifically, $71 \%(\mathrm{n}=15)$ were exposed to thiopurines in the last 3 months prior to the diagnosis of haematopoietic malignancy, which was significantly higher compared to patients who developed solid tumours ( $\mathrm{p}=0.041)(69)$.

Therefore, the current evidence does suggest that there is an increased relative risk of developing haematopoietic malignancy from the use of thiopurines. The absolute risk of developing these malignancies, however, remains low, specifically in patients without additional risk factors such as a young age in male patients, negative Epstein-Barr virus serology and prolonged exposure to the drug $(3,60-69)$.

\section{Non haematologic malignancies}

Finally, an association between thiopurines and NMSC risk have been demonstrated in patients with IBD (70). A meta-analysis showed that the risk of developing NMSCs with thiopurine use in IBD adult patients is nearly 2 -fold when only population-based studies were analysed (71). A recent retrospective cohort study in 2053 young patients with IBD, [median age at IBD diagnosis= 31.1 years (range 2.4-80.6 years) and median duration of illness per patient $=9.8$ years (range 1 month-19.6 years) $]$ showed that exposure to thiopurines $(\mathrm{OR}$ : 5.26, 95\% CI: $2.15-12.93, \mathrm{P}<0.001)$ and in particular thiopurines and/or TNF- $\alpha$ inhibitors (OR: 6.45, 95\% CI: 2.69-15.95, $\mathrm{P}<0.001$ ) was significantly associated with the development of NMSC. The majority (82\%) of patients exposed to a TNF- $\alpha$ inhibitor also had thiopurine exposure (72). In contrast to the risk of developing lymphoma, both ongoing and past exposure to thiopurines appears to significantly increase the risk of developing NMSC in patients with IBD (70). 


\section{Conclusions}

In IBD, including after acute severe colitis, and $\mathrm{AIH}$, thiopurines have proven their value as steroid sparing agents for the maintenance of remission. Their use with infliximab therapy reduces antibody formation and increases drug levels of the latter. More recently, however, the clinical use of thiopurines has been questioned due to their potential adverse effects as well as reduced efficacy when compared to new drugs, such as biologics. Both dose-independent and dependent side effects have been reported. Among dose-independent events, potential idiosyncratic or allergic reactions include rash, fever, arthralgias, pancreatitis, and hepatitis. The dose-dependent toxicities of thiopurines are mainly explained by their complex metabolism. Hepatotoxicity (associated with high 6MMP levels) and myelotoxicity (associated with high 6TGN and possibly 6MMP levels) are considered dosedependent reactions. Therefore, whenever thiopurine use is considered it is mandatory to first assess the risk/benefit ratio. The TPMT assay (either phenotype or genotype) is encouraged as a means to predict and therefore prevent life-threatening leukopenia. However, although TPMT genotype-phenotype correlation is high, this is not completely reliable. For this reason, periodic blood count monitoring, as well as liver and pancreas enzyme monitoring, remains essential throughout the duration of thiopurine therapy. Finally, although consideration should be given to the fact that thiopurine use does increase the incidence of specific severe malignancies (lymphoma, NMSCs, and hematologic malignancies), the absolute risk of their development is low.

In conclusion, thiopurines, either as monotherapy or in combination with other drugs, may still offer distinct advantages for the treatment of conditions such as IBD and AIH. Their use, however, should be tempered by an awareness of their adverse effects as well the most effective means of monitoring for such effects and/or preventing or limiting their occurrence. 


\section{References}

1. Lega S, Bramuzzo M, Dubinsky MC. Therapeutic Drug Monitoring in Pediatric IBD: Current Application and Future Perspectives. Curr Med Chem 2018; 25:2840-2854

2. Sahasranaman S, Howard D, Roy S. Clinical pharmacology and pharmacogenetics of thiopurines. Eur J Clin Pharmacol 2008; 64:753-67.

3. Hyams JS, Dubinsky MC, Baldassano RN, et al. Infliximab is not associated with increased risk of malignancy or hemophagocytic lymphohistiocytosis in pediatric patients with inflammatory bowel disease. Gastroenterology 2017;152:1901-1914.e3.

4. Wilson DC, Griffiths AM. Thiopurine Monotherapy in Pediatric Inflammatory Bowel Disease: 20 Years After Markowitz. J Pediatr Gastroenterol Nutr 2020; [Epub ahead of print]

5. Beswick L, Friedman AB, Sparrow MP. The role of thiopurine metabolite monitoring in inflammatory bowel disease. Expert Rev Gastroenterol Hepatol 2014;8:383-92.

6. Lennard L. The clinical pharmacology of 6-mercaptopurine. Eur J Clin Pharmacol 1992, 43: 329-339.

7. Thomas CW, Myhre GM, Tschumper R, et al. Selective inhibition of inflammatory gene expression in activated T lymphocytes: a mechanism of immune suppression by thiopurines. J Pharmacol Exp Ther 2005;312:537-545.

8. Iede I, Fritz G, Strand Set al. CD28-dependent Rac1 activation is the molecular target of azathioprine in primary human CD4+ T lymphocytes. J Clin Invest 2003;111:113345.

9. Ruemmele FM, Veres G, Kolho KL, et al. Consensus guidelines of ECCO/ESPGHAN on the medical management of pediatric Crohn's disease. $J$ Crohn's Colitis 2014; 8: 1179-1207. 
10. Turner D, Ruemmele FM, Orlanski-Meyer E, et al. Management of Paediatric Ulcerative Colitis, Part 1:Ambulatory Care-An Evidence-based Guideline From European Crohn's and ColitisOrganization and European Society of Paediatric Gastroenterology, Hepatology and Nutrition. J Pediatr Gastroenterol Nutr 2018;67:257-291.

11. Mack DR, Benchimol EI, Critch J, et al. Canadian Association of Gastroenterology Clinical Practice Guideline for the Medical Management of Pediatric Luminal Crohn's Disease. Gastroenterology 2019; 157: 320-348.

12. Turner D, Ruemmele FM, Orlanski-Meyer E, et al. Management of Paediatric Ulcerative Colitis, Part 2: Acute Severe Colitis-An Evidence-based Consensus Guideline From the European Crohn's and Colitis Organization and the European Society of Paediatric Gastroenterology, Hepatology and Nutrition. J Pediatr Gastroenterol Nutr 2018; 67: 292-310.

13. Amil-Dias J, Kolacek S, Turner D, et al. Surgical Management of Crohn Disease in Children: Guidelines From the Paediatric IBD Porto Group of ESPGHAN. J Pediatr Gastroenterol Nutr 2017; 64:818-835.

14. Doherty G, Bennett G, Patil S, et al. Interventions for prevention of post-operative recurrence of Crohn's disease. Cochrane Database Syst Rev 2009;4:CD006873;

15. Papay P, ReinischW, Ho E, et al. The impact of thiopurines on the risk of surgical recurrence in patients with Crohn's disease after first intestinal surgery. Am $J$ Gastroenterol 2010;105:1158-64

16. Atia O, Ledder O, Ben-Moshe T, et al. Role of Thiopurines in Pediatric Inflammatory Bowel Diseases: A Real-Life Prospective Cohort Study. J Pediatr Gastroenterol Nutr 2020; [Epub ahead of print] 
17. Colombel JF, Adedokun OJ, Gasink C, et al. Infliximab, azathioprine, or combination therapy for Crohn's disease. N Engl J Med 2010;362:1383-1395.

18. Panaccione R, Ghosh S, Middleton S, et al. Combination therapy with infliximab and azathioprine is superior to monotherapy with either agent in ulcerative colitis. Gastroenterology 2014;146:392-400.

19. Kansen HM, van Rheenen PF, Houwen RHJ, et al. Kids with Crohn's, Colitis (KiCC) Working Group for Collaborative Paediatric IBD Research in the Netherlands. Less anti-infliximab antibody formation in paediatric crohn patients on concomitant immunomodulators. J Pediatr Gastroenterol Nutr 2017;65:425-429.

20. Singh N, Rosenthal CJ, Melmed GY, et al Early infliximab trough levels are associated with persistent remission in pediatric patients with inflammatory bowel disease. Inflamm Bowel Dis 2014; 20:1708-1713.

21. Chi, LY, Zitomersky, NL, Liu E, et al.. The impact of combination therapy on infliximab levels and antibodies in children and young adults with inflammatory bowel disease. Inflamm Bowel Dis 2018, 24, 1344-1351.

22. Kierkus, J, Iwanczak, B, Wegner, A, et al. Monotherapy with infliximab versus combination therapy in the maintenance of clinical remission in children with moderate to severe Crohn disease. J Pediatr Gastroenterol Nutr 2015;60:580-585.

23. Roblin X, Boschetti G, Williet N, et al. Azathioprin dose reduction in inflammatory bowel disease patients on combination therapy: an open-label, prospective and randomised clinical trial. Aliment Pharmacol Ther 2017; 46:142-149.

24. Yarur AJ, Kubiliun MJ, Czul F, et al. Concentrations of 6-thioguanine nucleotide correlate with trough levels of infliximab in patients with inflammatory bowel disease on combination therapy. Clin Gastroenterol Hepatol 2015; 13: 1118-24.e3. 
25. Kennedy NA, Heap GA, Green HD, et al. UK Inflammatory Bowel Disease Pharmacogenetics Study Group. Predictors of anti-TNF treatment failure in anti-TNFnaive patients with active luminal Crohn's Disease: a prospective, multicentre, cohort study. Lancet Gastroenterol Hepato 2019; 4 :341-353.

26. Colombel JF, Reinisch W, Mantzaris GJ, et al. Randomised clinical trial: deep remission in biologic and immunomodulator naïve patients with Crohn's disease - $\mathrm{a}$ SONIC post hoc analysis. Aliment Pharmacol Ther 2015; 41: 734-46.

27. Terziroli Beretta-Piccoli B, Mieli-Vergani G, et al. Autoimmune hepatitis: Standard treatment and systematic review of alternative treatments. World J Gastroenterol 2017; 23:6030-6048.

28. Sheiko MA, Sundaram SS, Capocelli, KE, et al. Outcomes in Pediatric Autoimmune Hepatitis and Significance of Azathioprine Metabolites. J Ped Gastroenterol Nutr 2017;65: 80-85.

29. Mieli-Vergani G, Vergani D, Baumann U, et al. Diagnosis and Management of Pediatric Autoimmune Liver Disease: ESPGHAN Hepatology Committee Position Statement. J Pediatr Gastroenterol Nutr 2018 ;66:345-360.

30. Gordon M, Taylor K, Akobeng AK, et al. Azathioprine and 6-mercaptopurine for maintenance of surgically-induced remission in Crohn's disease. Cochrane Database Syst Rev 2014;8:CD010233.

31. Grossman AB, Noble AJ, Mamula P, et al. Increased dosing requirements for 6mercaptopurine and azathioprine in inflammatory bowel disease patients six years and younger. Inflamm Bowel Dis 2008;14:750-5.

32. Lennard L. Assay of 6-thioinosinic acid and 6-thioguanine nucleotides, active metabolites of 6-mercaptopurine, in human red blood cells. $J$ Chromatogr 1987;423:169-78. 
33. Dubinsky MC, Lamothe S, Yang HY, et al. Pharmacogenomics and metabolite measurement for 6-mercaptopurine therapy in inflammatory bowel disease. Gastroenterology, 2000,;118:705-713.

34. Hradsky O, Potuznikova K, Siroka J, et al Prediction of Thiopurine Metabolite Levels Based on Hematological and Biochemical Parameters. J Pediatr Gastroenterol Nutr 2019; 69:e105-e110.

35. Kandavel P, Eder SJ, Newman NE, et al. Mean Corpuscular Volume to White Blood Cell Ratio for Thiopurine Monitoring in Pediatric Inflammatory Bowel Disease. $J$ Pediatr Gastroenterol Nutr 2019; 69:88-94.

36. Spencer E, Norris E, Williams C, et al. The Impact of Thiopurine Metabolite Monitoring on the Durability of Thiopurine Monotherapy in Pediatric IBD. Inflamm Bowel Dis 2019; 25:142-149.

37. Weinshilboum RM, Sladek SL. Mercaptopurine pharmacogenetics: monogenic inheritance of erythrocyte thiopurine methyltransferase activity. Am J Hum Genet 1980;32:651-662.

38. Stocco G, Martelossi S, Arrigo S, et al. Multicentric case-control study on azathioprine dose and pharmacokinetics in early-onset pediatric inflammatory bowel disease. Inflamm Bowel Dis 2017;23: 628-634.

39. Lichtenstein GR, Abreu MT, Cohen R, et al. American Gastroenterological Association Institute medical position statement on corticosteroids, immunomodulators, and infliximab in inflammatory bowel disease. Gastroenterology 2006;130: 935-9.

40. Sluiter RL, Van Marrewijk C, De Jong D, et al. Genotype-Guided Thiopurine Dosing Does not Lead to Additional Costs in Patients With Inflammatory Bowel Disease. $J$ Crohns Colitis 2019;13: 838-845. 
41. Chang JY, Park SJ, Jung ES, et al. Genotype-based Treatment With Thiopurine Reduces Incidence of Myelosuppression in Patients With Inflammatory Bowel Diseases. Clin Gastroenterol Hepatol 2019;S1542-3565(19)30909-7.

42. Fan X, Yin D, Men R, et al. NUDT15 Polymorphism Confer Increased Susceptibility to Thiopurine-Induced Leukopenia in Patients With Autoimmune Hepatitis and Related Cirrhosis. Front Pharmacol 2019;10:346.

43. Koutsilieri S, Caudle KE, Alzghari SK, et al. Optimizing thiopurine dosing based on TPMT and NUDT15 genotypes: It takes two to tango. Am J Hematol 2019; 94:737740.

44. Ford LT, Berg JD. Thiopurine S-methyltransferase (TPMT) assessment prior to starting thiopurine drug treatment; a pharmacogenomic test whose time has come. $J$ Clin Pathol 2010; 63:288-95.

45. Mottet C, Schoepfer AM, Juillerat P, et al. Experts Opinion on the Practical Use of Azathioprine and 6-Mercaptopurine in Inflammatory Bowel Disease. Inflamm Bowel Dis 2016; 22: 2733-2747.

46. Timmer A, Patton PH, Chande N, et al. Azathioprine and 6-mercaptopurine for maintenance of remission in ulcerative colitis. Cochrane Database Syst Rev 2016;2016:CD000478.

47. Fuentes D, Torrente F, Keady S, et al. High-dose azathioprine in children with inflammatory bowel disease. Aliment Pharmacol Ther 2003;17:913-21.

48. Kirschner BS. Safety of azathioprine and 6-mercaptopurine in pediatric patients with inflammatory bowel disease. Gastroenterology 1988; 115:813-821.

49. Kennedy NA, Rhatigan E, Arnott ID, et al. A trial of mercaptopurine is a safe strategy in patients with inflammatory bowel disease intolerant to azathioprine: an 
observational study, systematic review and meta-analysis. Aliment Pharmacol Ther 2013;38: 1255-66

50. McLean LP, Cross RK. Adverse events in IBD: to stop or continue immune suppressant and biologic treatment. Expert Rev Gastroenterol Hepatol 2014;8:223-40.

51. Chaparro M, Ordás I, Cabré E, et al. Safety of thiopurine therapy in inflammatory bowel disease: long-term followup study of 3931 patients. Inflamm Bowel Dis 2013;19:1404-10.

52. Weersma RK, Peters FT, Oostenbrug LE, et al. Increased incidence of azathioprineinduced pancreatitis in Crohn's disease compared with other diseases. Aliment Pharmacol Ther 2004; 20:843-50.

53. Wintzell V, Svanström H, Olén O, et al. Association between use of azathioprine and risk of acute pancreatitis in children with inflammatory bowel disease: a SwedishDanish nationwide cohort study. Lancet Child Adolesc Health 2019;3:158-165..

54. Toruner M, Loftus EV Jr, Harmsen WS, et al. Risk factors for opportunistic infections in patients with inflammatory bowel disease. Gastroenterology 2008;134:929-36.

55. Kirchgesner J, Lemaitre M, Carrat F, et al. Risk of Serious and Opportunistic Infections Associated With Treatment of Inflammatory Bowel Diseases. Gastroenterology 2018;155:337-346.e10.

56. Hyams JS, Dubinsky M, Rosh J, et al. The effects of concomitant immunomodulators on the pharmacokinetics, efficacy and safety of adalimumab in paediatric patients with Crohn's disease: a post hoc analysis. Aliment Pharmacol Ther 2019; 49:155-164.

57. Musumba CO. Review article: the association between nodular regenerative hyperplasia, inflammatory bowel disease and thiopurine therapy. Aliment Pharmacol Ther 2013; 38: 1025-1037 
58. Kreijne JE, de Veer RC, de Boer NK, et al. Real-life study of safety of thiopurineallopurinol combination therapy in inflammatory bowel disease: myelotoxicity and hepatotoxicity rarely affect maintenance treatment. Aliment Pharmacol Ther 2019;50:407-415.

59. Dayharsh GA, Loftus EV, Sandborn WJ, et al. EBV-positive lymphoma in patients with inflammatory bowel disease treated with AZA or 6-mercaptopurine. Gastroenterology 2002;122:72-7

60. Beaugerie L, Brousse N, Bouvier AM, et al. Lymphoproliferative disorders in patients receiving thiopurines for inflammatory bowel disease: a prospective observational cohort study. Lancet 2009; 374:1617-25.

61. Vos AC, Bakkal N, Minnee RC, et al. Risk of malignant lymphoma in patients with inflammatory bowel diseases: a Dutch nationwide study. Inflamm Bowel Dis 2011;17:1837-45.

62. Rahier JF, Magro F, Abreu C, et al. Second European evidence-based consensus on the prevention, diagnosis and management of opportunistic infections in inflammatory bowel disease. J Crohns Colitis 2014; 8: 443-68.

63. Martinelli M, Giugliano FP, Strisciuglio C, et al. Vaccinations and Immunization Status in Pediatric Inflammatory Bowel Disease: A Multicenter Study From the Pediatric IBD Porto Group of the ESPGHAN. Inflamm Bowel Dis 2019 Nov 5. [Epub ahead of print]

64. Kotlyar DS, Lewis JD, Beaugerie L, et al. Risk of lymphoma in patients with inflammatory bowel disease treated with azathioprine and 6-mercaptopurine: a metaanalysis. Clin Gastroenterol Hepatol 2015;13:847-858 e4; quiz e48-50. 
65. Lemaitre M, Kirchgesner J, Rudnichi A, et al. Association between use of thiopurines or tumor necrosis factor antagonists alone or in combination and risk of lymphoma in patients with inflammatory bowel disease. JAMA 2017;318:1679-1686

66. http://seer.cancer.gov/csr/1975_2007/results_merged/sect_09_hodgkins.pdf. 2010. National Cancer Institute

67. http: //seer.cancer.gov/csr/1975_2007/results_merged/sect_19_nhl.pdf. 2010

68. Kotlyar D, Gisbert J, Chaparro M, et al. Demographic Difference of Hepatosplenic TCell Lymphoma (HSTCL) Versus Non-HSTCL Lymphoma in Patients with IBD Receiving Thiopurines: A Population Cohort Analysis. American Journal of Gastroenterology 2012;107:S632

69. Joosse ME, Aardoom MA, Kemos P, et al. Paediatric IBD Porto group of ESPGHAN. Malignancy and mortality in paediatric-onset inflammatory bowel disease: a 3-year prospective, multinational study from the paediatric IBD Porto group of ESPGHAN. Aliment Pharmacol Ther 2018;48:523-537.

70. Peyrin-Biroulet L, Khosrotehrani K, Carrat F, et al. Increased risk for nonmelanoma skin cancers in patients who receive thiopurines for inflammatory bowel disease. Gastroenterology 2011;141:1621-1628. e1-5.

71. Ariyaratnam J, Subramanian V. Association between thiopurine use and nonmelanoma skin cancers in patients with inflammatory bowel disease: a metaanalysis. Am J Gastroenterol 2014;109:163-169.

72. Clowry J, Sheridan J, Healy R, et al. Increased non-melanoma skin cancer risk in young patients with inflammatory bowel disease on immunomodulatory therapy: a retrospective single-centre cohort study. J Eur Acad Dermatol Venereol 2017;31:978985. 


\section{Figure 1 Legend}

Metabolic pathways of Azathioprine

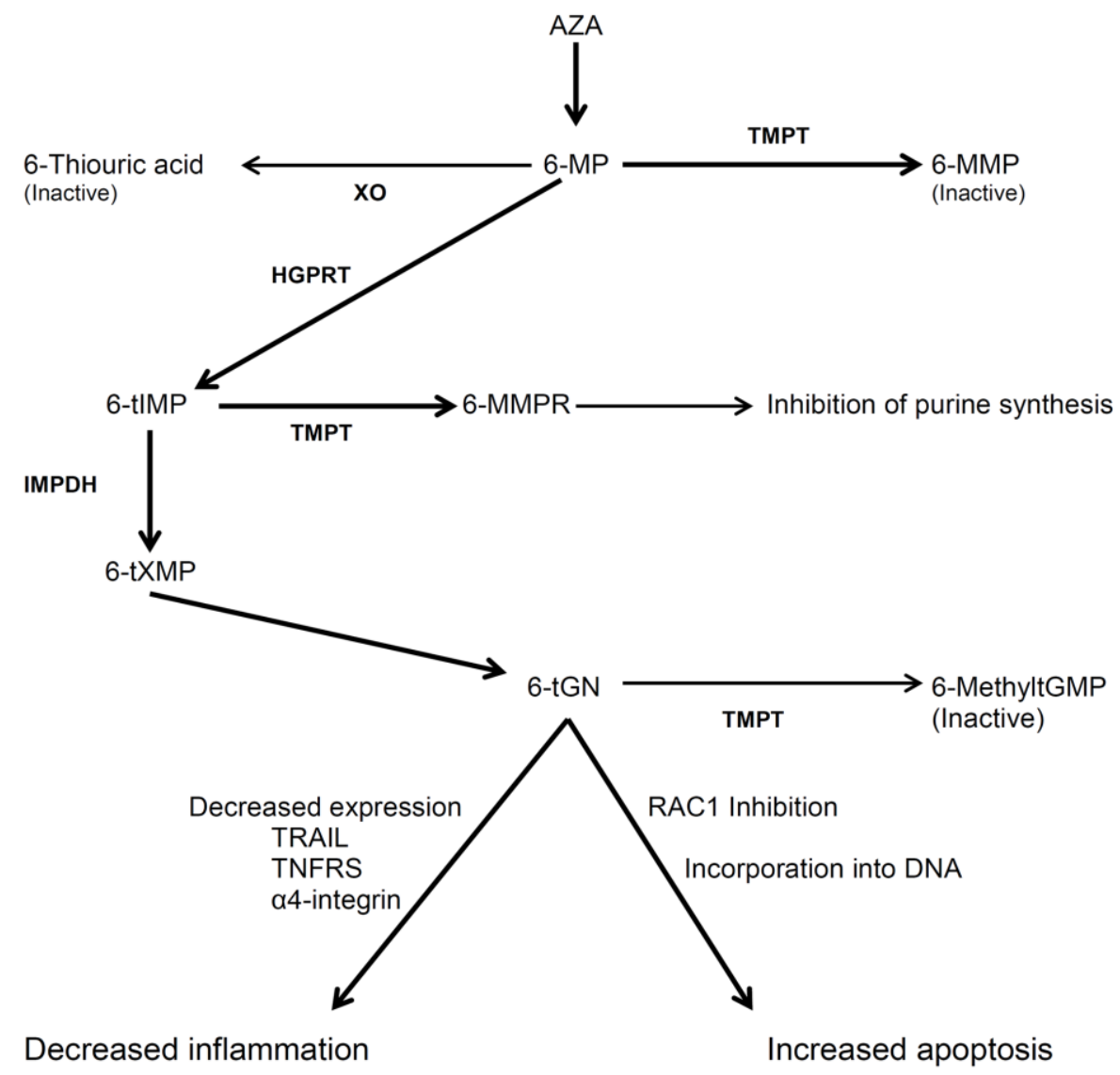

Abbreviations: $\mathrm{AZA}=$ azathioprine; $\mathrm{XO}=$ xanthine oxidase; $6-\mathrm{MP}=6$-mercaptopurine; 6 MMP: 6-Methylmercaptopurine; $\quad$ TPMT = thiopurine S-methyltransferase; HGPRT = hypoxanthine guanine phosphoribosyltransferase; 6-tIMP $=$ 6-thiomercaptopurine; 6MMPR: 6-Methylmercaptopurine ribonucleotide; $\mathrm{IMPDH}=$ inosine monophosphate dehydrogenase; 6-tXMP = 6-thioxanthosine; 6-tGN = 6-thioguanine nucleotides; 6MethyltGMP =thioguanine monophosphate. 
Table 1. Prevalence in the General Population of Adverse Effects Associated with Thiopurine Therapy

\begin{tabular}{|c|c|c|c|c|}
\hline Adverse effect & Type & Incidence & When & Action \\
\hline Leukopenia & $\mathrm{DD}$ & $10 \%$ & Anytime & CBC, $(6-T G N)$ \\
\hline Agranulocytosis & DD & $0.3 \%$ & Within $4-8 \mathrm{wk}$ & CBC, (TPMT activity) \\
\hline $\begin{array}{l}\text { Gastrointestinal } \\
\text { intolerance }\end{array}$ & $\mathrm{DD} / \mathrm{DI}$ & $5 \%-20 \%$ & Within 6-8 wk & Patient information \\
\hline Hepatitis & DI & $15 \%-28 \%$ & $\begin{array}{l}\text { Anytime but } \\
\text { increases gradually } \\
\text { over time }\end{array}$ & LFTs, (6-MMP) \\
\hline Pancreatitis & DI & $3 \%-15 \%$ & Within 3 mo & Lipase \\
\hline Flu-like illness & DI & $5 \%$ & Within $2 \mathrm{wk}$ & $\begin{array}{l}\text { Patient information, body } \\
\text { temnerature }\end{array}$ \\
\hline Rash & DI & $4 \%$ & Within $3 \mathrm{wk}$ & Patient information \\
\hline
\end{tabular}

DD: dose dependent; DI: dose independent CBC, complete blood count; LFT, liver function test 